\title{
Accelerated protein engineering using Vibrio natriegens genetic code expansion
}

\author{
Eden Ozer $^{1}$ and Lital Alfonta ${ }^{2}$ \\ ${ }^{1}$ Ben Gurion University of the Negev \\ ${ }^{2}$ Ben Gurion University of the Negev
}

July 7, 2020

\begin{abstract}
Escherichia coli has been considered as the most used model bacteria in the majority of studies for several decades. However, a new faster chassis is emerging in the form of the fast-growing gram-negative bacterium Vibrio natriegens. Different methodologies, well established in E. coli, are currently being adapted for the Vibrio natriegens in the hope of enabling a much faster platform for general lab-work. Amongst the vast technologies available for E. coli, genetic code expansion, the incorporation of unnatural amino acids into proteins, serves as a robust tool for protein engineering and biorthogonal modifications. Here we designed and adapted the genetic code expansion methodology for Vibrio natriegens and demonstrate an unnatural amino acid incorporation into a protein for the first time in this organism.
\end{abstract}

\section{Hosted file}

V.nat V4.docx available at https://authorea.com/users/339976/articles/467728-acceleratedprotein-engineering-using-vibrio-natriegens-genetic-code-expansion 